\title{
Correlating Cryo-Electron Microscopy Methods for Structural Studies of Bacteria.
}

Elizabeth R. Wright ${ }^{1,2}$, Hong $\mathrm{Yi}^{2}$, Jeannette V. Taylor ${ }^{2}$, Ricardo C. Guerrero-Ferreira ${ }^{1}$ and Cheri M. Hampton $^{1}$

1. Emory University School of Medicine, Division of Pediatric Infectious Disease, Children's Healthcare of Atlanta, Atlanta, Georgia

2. Robert P. Apkarian Integrated Electron Microscopy Core, Emory University, Atlanta, Georgia

Vibrio vulnificus, a halotrophic Gram-negative bacterium, which lives in coastal waters, is associated with shellfish in natural environments and is an opportunistic pathogen of humans. A number of studies have identified many of the virulence factors associated with $V$. vulnificus [1]. Some of the virulence factors identified include external structures of the bacteria such as the capsular polysaccharide (CPS), flagellum, and pili. However, limited efforts have been aimed at determining the ultrastructure of these factors and the arrangement of their individual components. In order to address how ultrastructure of the bacteria influences pathogenesis, we probed the architecture of the cells, the flagella, the pili, and the CPS with high-resolution scanning electron microscopy (HRSEM), conventional transmission electron microscopy (TEM) of cryo-preserved specimens, and cryo-electron tomography (cryo-ET).

We used HRSEM methods to examine the structure of the bacterial outer membrane, appendages, and secreted outer membrane vesicles (OMVs). V. vulnificus cultures were fixed and then dehydrated with increasing concentrations of ethanol. Samples were dried in a Polaron critical point drying (CPD) apparatus. The specimens were mounted onto stubs and a thin film of chromium was deposited with a Denton DV-602 magnetron sputter coater. Specimens were imaged in ISI DS-130F FE-SEM.

We used a combination of cryo-preservation methods and conventional TEM imaging to assess the presence or absence of the bacterial capsular polysaccharide (CPS) layer and whether secreted OMVs remained associated with the cell. V. vulnificus strains were cultured overnight either on agar plates or in liquid media. Bacteria grown on plates were either used as individual colonies or gently scraped together to produce a larger volume of material. The individual colonies or the pooled sample were applied to Balzers planchets and high pressure frozen (HPF) with a Balzers HPM 010. Alternatively, the pooled sample was pipetted into Leica copper capillary tubes, the tube ends were crimped, and subsequently frozen in liquid propane by self-pressurized rapid freezing (SPRF). The frozen hydrated samples were freeze substituted using a Leica EM AFS2 automatic freeze substitution system. Resin-infiltrated samples were sectioned, post-stained and imaged on a JEOL JEM-1400 120 kV LaB 6 -TEM.

Liquid cultures of $V$. vulnificus strains were aliquoted onto glow-discharged Quantifoil carbon grids and then plunge-frozen in liquid ethane with a FEI Mark III Vitrobot. Cryo-EM and cryo-ET data collection was performed with a JEOL JEM-2200FS $200 \mathrm{kV} \mathrm{FEG-TEM} \mathrm{equipped} \mathrm{with} \mathrm{the} \mathrm{Zernike} \mathrm{phase} \mathrm{plate}$ airlock system, an in-column energy filter (slit width $20 \mathrm{eV}$ ), a cryo-transfer specimen holder (Model 914, Gatan), a DE-20 direct electron detection device (Direct Electron, LP), and a 4k x 4k Gatan Ultrascan CCD camera. Tilt series were acquired automatically with $2^{\circ}$ tilt increments from $-65^{\circ}$ to $+65^{\circ}$ by using Serial EM [2]. Reconstructions were generated with IMOD [3].

HRSEM studies resolved the overall cell structure (Figure 1). Rapid cryo-preservation techniques combined with TEM imaging resolved the presence of the CPS extending from the cell surface (Figure 2). In addition, OMVs were observed within the CPS matrix. Cryo-ET data provided information 
regarding the three-dimensional (3D) arrangement of released OMVs (Figure 3). Studies are underway to improve bacterial preservation and imaging methods of the $V$. vulnificus CPS as well as OMV order.

\section{References:}

[1] PA Gulig, KL Bourdage, and AM Starks, J. Microbiology 43 (2005), p. 118-131.

[2] DN Mastronarde, J. Structural Biology 152(1) (2005), p. 36-51.

[3] JR Kremer, DN Mastronarde, and JR McIntosh, J. Structural Biology 116(1) (1996), p. 71-76.

[4] This research was supported by funds from Emory University, Children's Healthcare of Atlanta, the Emory Center for AIDS Research, the Georgia Research Alliance, Human Frontiers Science Program, National Institutes of Health (1R01GM104540 and 1R21AI101775), and the National Science Foundation (0923395) to E.R.W, and S10 RR025679 to P.W.S. Cryo-EM/ET data was collected at the Emory University Robert P. Apkarian Integrated Electron Microscopy Core.

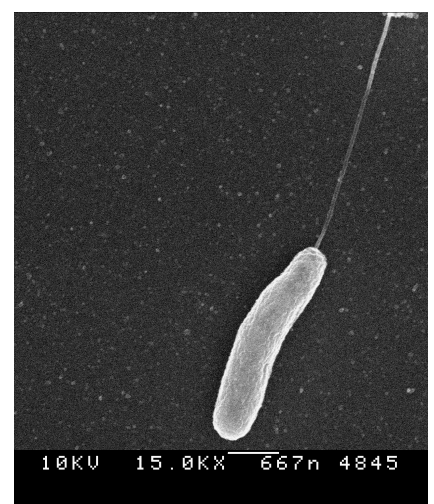

Figure 1. FE-SEM images of a $V$. vulnificus cells. Scale bar, $667 \mathrm{~nm}$.

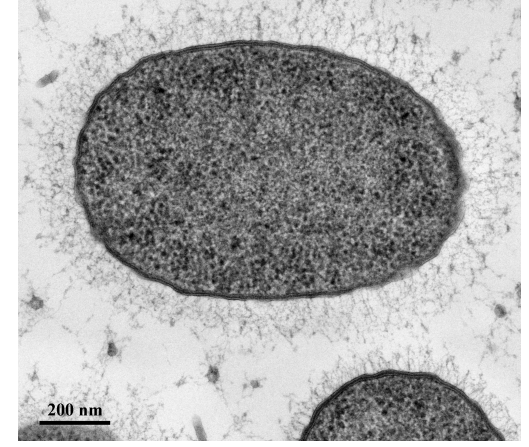

Figure 2. Conventional TEM images of SPRF preserved V. vulnificus cell. Scale bar, $200 \mathrm{~nm}$.

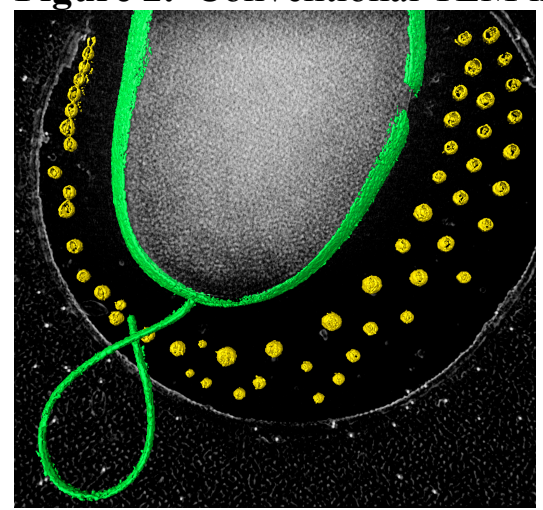

Figure 3. Segmented 3D reconstruction of a frozen-hydrated $V$. vulnificus cell. 